\title{
Intrabeam scattering formulas for fast numerical evaluation
}

\author{
Sergei Nagaitsev \\ FNAL, P.O. Box 500, Batavia, Illinois 60510, USA \\ (Received 1 April 2005; published 30 June 2005)
}

\begin{abstract}
Small-angle multiple intrabeam scattering (IBS) emittance growth rates are normally expressed through integrals, which require a numeric evaluation at various locations of the accelerator lattice. In this paper, I demonstrate that the IBS growth rates can be presented in closed-form expressions with the help of the socalled symmetric elliptic integral. This integral can be evaluated numerically by a very efficient recursive method by employing the duplication theorem. Several examples of IBS rates for a smooth-lattice approximation, equal transverse temperatures and plasma temperature relaxation are given.
\end{abstract}

DOI: $10.1103 /$ PhysRevSTAB.8.064403

PACS numbers: 29.20. $-\mathrm{c}$, 29.27.Fh, 52.25.Dg

\section{INTRODUCTION}

This paper presents the results, previously obtained by Bjorken and Mtingwa [1], as closed-form analytic expressions. In fact, all of the rates, presented here, are strictly identical to the ones in Ref. [1]. Also, the notations are essentially the same as in Ref. [1]. Suppose that the bunched-beam distribution function, $f$, is described by the following expression:

$$
f=\exp (-S)
$$

where

$$
S=\frac{1}{2} A_{\alpha \beta} \frac{p_{\alpha}}{p} \frac{p_{\beta}}{p}+\frac{1}{2} B_{\alpha \beta} x_{\alpha} \frac{p_{\beta}}{p}+\frac{1}{2} C_{\alpha \beta} x_{\alpha} x_{\beta},
$$

$x_{\alpha}(=x, y$, or $s)$ is the particle coordinate, $p_{\alpha}$ is its momentum and $p=\beta \gamma M c$. It is normalized to a total 6dimensional $\mathrm{rms}$ invariant phase-space volume, $\Gamma$, as

$$
\Gamma=\int f d^{3} x d^{3} p
$$

There have been attempts in the past [1,2] to express the intrabeam scattering (IBS) rates through Legendre's incomplete elliptic integrals. Let me now introduce a symmetric elliptic integral of the second kind, $R_{D}(x, y, z)$, following Carlson's definition [3]

$$
R_{D}(x, y, z)=\frac{3}{2} \int_{0}^{\infty} \frac{d t}{\sqrt{(t+x)(t+y)(t+z)^{3}}} .
$$

The following are some useful properties of this integral:

$$
\begin{gathered}
R_{D}(x, x, x)=x^{-3 / 2}, \\
R_{D}(x, y, z)+R_{D}(y, z, x)+R_{D}(z, x, y)=\frac{3}{\sqrt{x y z}},
\end{gathered}
$$

and

$$
R_{D}(h x, h y, h z)=h^{-3 / 2} R_{D}(x, y, z) \quad(\text { for } h>0) .
$$

There exists the so-called duplication theorem [4] for this integral, which allows for a very efficient numerical evalu- ation using a recursive method [5]. Only rational operations and square roots are required. Such a numerical method gives, in my opinion, the main advantage for expressing the IBS rates through this integral.

The IBS formulas, I am proposing in this paper, require evaluating the symmetric elliptic integral, with its variables cycled, 3 times at each point of the accelerator lattice. Actually, Eq. (6) allows us to reduce the number of integrals to two.

Some of the IBS rates for special cases are expressed by the following combination of elliptic integrals:

$\Psi(x, y, z)=-2 x R_{D}(y, z, x)+y R_{D}(z, x, y)+z R_{D}(x, y, z)$.

For example, Eq. (3.6) in B-M [1] paper can be written as follows:

$$
\begin{aligned}
\frac{1}{\tau} \equiv & \frac{1}{\Gamma} \frac{d \Gamma}{d t} \\
= & \frac{2 \pi^{2} N c r_{p}{ }^{2} M^{3} L_{C}}{3 \gamma \Gamma} \int_{0}^{L} \frac{d s}{L \sqrt{\lambda_{1} \lambda_{2} \lambda_{3}}}\left[\lambda_{1} \Psi\left(\frac{1}{\lambda_{1}}, \frac{1}{\lambda_{2}}, \frac{1}{\lambda_{3}}\right)\right. \\
& + \text { two cyclic permutations }],
\end{aligned}
$$

where $\lambda_{1}, \lambda_{2}$, and $\lambda_{3}$ are the eigenvalues of the matrix $\boldsymbol{A}$ in Eq. (2), $N$ is the number of particles per bunch, $L_{C}$ is the Coulomb $\log , \ln \left(b_{\max } / b_{\min }\right)$ with $b_{\max }$ and $b_{\min }$ being the maximum and minimum impact parameters as defined in Ref. [1], $c$ is the speed of light, $M$ is the particle mass, $r_{p}$ is the classical particle radius, and $L$ is the ring circumference.

\section{IBS RATES FOR UNCOUPLED BETATRON OSCILLATIONS}

Uncoupled betatron oscillations can be described by the following expressions:

$$
x=\sqrt{2 J_{x} \beta_{x}} \cos \left(\varphi_{x}\right)+D_{x} \delta,
$$




$$
\begin{gathered}
\frac{p_{x}}{p}=x^{\prime}=D_{x}^{\prime} \delta+\frac{\beta_{x}^{\prime}}{2 \beta_{x}}\left(x-D_{x} \delta\right)-\sqrt{\frac{2 J_{x}}{\beta_{x}}} \sin \left(\varphi_{x}\right), \\
y=\sqrt{2 J_{y} \beta_{y}} \cos \left(\varphi_{y}\right), \\
\frac{p_{y}}{p}=y^{\prime}=\frac{\beta_{y}^{\prime}}{2 \beta_{y}} y-\sqrt{\frac{2 J_{y}}{\beta_{y}}} \sin \left(\varphi_{y}\right),
\end{gathered}
$$

where

$$
J_{x}=\frac{1}{2 \beta_{x}}\left(x-D_{x} \delta\right)^{2}+\frac{\beta_{x}}{2}\left(x^{\prime}-\frac{\beta_{x}^{\prime}}{2 \beta_{x}} x-\Phi \delta\right)^{2}
$$

and

$$
J_{y}=\frac{y^{2}}{2 \beta_{y}}+\frac{\beta_{y}}{2}\left(y^{\prime}-\frac{\beta_{y}^{\prime}}{2 \beta_{y}} y\right)^{2}
$$

are the action variables, $\varphi_{x}, \varphi_{y}$ are the betatron phase variables of unperturbed oscillations, and $\delta$ is the relative laboratory frame momentum spread. The function $\Phi$ is expressed as follows:

$$
\Phi=D_{x}^{\prime}-\frac{\beta_{x}^{\prime} D_{x}}{2 \beta_{x}} .
$$

Similarly, the synchrotron action variable for a parabolic potential well is described by

$$
J_{s}=\frac{s^{2}}{2 k_{s}}+\frac{k_{s}}{2} \delta^{2} .
$$

Suppose now that the distribution function, $f$, in Eq. (1) can be written as

$$
f=\exp \left[-\frac{J_{x}}{\varepsilon_{x}}-\frac{J_{y}}{\varepsilon_{y}}-\frac{J_{s}}{\varepsilon_{s}}\right],
$$

where $\varepsilon_{x} \varepsilon_{y}$ are the rms non-normalized transverse emittances, and $\varepsilon_{s}=\sigma_{p} \sigma_{s}$ with $\sigma_{s}$ and $\sigma_{p}$ being the rms bunch length and the rms relative lab-frame momentum spread.

Before presenting the IBS rate formulas, I will first define several parameters. Let

$$
\begin{gathered}
a_{x}=\frac{\beta_{x}}{\varepsilon_{x}} \equiv \frac{1}{\theta_{x}^{2}}, \quad a_{y}=\frac{\beta_{y}}{\varepsilon_{y}} \equiv \frac{1}{\theta_{y}^{2}}, \\
\sigma_{x}=\sqrt{D_{x}^{2} \sigma_{p}^{2}+\varepsilon_{x} \beta_{x}}, \quad \sigma_{y}=\sqrt{\varepsilon_{y} \beta_{y}}, \\
a_{s}=a_{x}\left(\frac{D_{x}^{2}}{\beta_{x}^{2}}+\Phi^{2}\right)+\frac{1}{\sigma_{p}^{2}},
\end{gathered}
$$

and

$$
a_{1}=\frac{1}{2}\left(a_{x}+\gamma^{2} a_{s}\right), \quad a_{2}=\frac{1}{2}\left(a_{x}-\gamma^{2} a_{s}\right) .
$$

The three eigenvalues of the matrix $\boldsymbol{A}$ [Eq. (2)] can be now expressed as follows:

$$
\begin{gathered}
\lambda_{1}=a_{y}, \\
\lambda_{2}=a_{1}+\sqrt{a_{2}{ }^{2}+\gamma^{2} a_{x}{ }^{2} \Phi^{2}}, \\
\lambda_{3}=a_{1}-\sqrt{a_{2}{ }^{2}+\gamma^{2} a_{x}{ }^{2} \Phi^{2}} .
\end{gathered}
$$

The three integrals are calculated at each location of the ring lattice as follows:

$$
\begin{aligned}
& R_{1}=\frac{1}{\lambda_{1}} R_{D}\left(\frac{1}{\lambda_{2}}, \frac{1}{\lambda_{3}}, \frac{1}{\lambda_{1}}\right), \\
& R_{2}=\frac{1}{\lambda_{2}} R_{D}\left(\frac{1}{\lambda_{3}}, \frac{1}{\lambda_{1}}, \frac{1}{\lambda_{2}}\right), \\
& R_{3}=\frac{1}{\lambda_{3}} R_{D}\left(\frac{1}{\lambda_{1}}, \frac{1}{\lambda_{2}}, \frac{1}{\lambda_{3}}\right) .
\end{aligned}
$$

Here, Eq. (7) can be used to avoid extreme values in the arguments of $R_{D}(x, y, z)$ and Eq. (6) to reduce the number of integrals to two.

The total 6-dimensional emittance growth rate in Eq. (9) can be written as

$$
\begin{aligned}
\frac{1}{\tau} \equiv & \frac{1}{\Gamma} \frac{d \Gamma}{d t}=\frac{1}{\varepsilon_{x}} \frac{d \varepsilon_{x}}{d t}+\frac{1}{\varepsilon_{y}} \frac{d \varepsilon_{y}}{d t}+\frac{1}{\sigma_{p}^{2}} \frac{d \sigma_{p}^{2}}{d t} \\
= & \frac{N r_{p}{ }^{2} c L_{C}}{12 \pi \beta^{3} \gamma^{5} \sigma_{s}} \int_{0}^{L} \frac{d s}{L \sigma_{x} \sigma_{y}}\left[\lambda_{1} \Psi\left(\frac{1}{\lambda_{1}}, \frac{1}{\lambda_{2}}, \frac{1}{\lambda_{3}}\right)\right. \\
& + \text { two cyclic permutations }],
\end{aligned}
$$

where

$$
\Gamma=8 \pi^{3} \beta^{3} \gamma^{3} M^{3} c^{3} \varepsilon_{x} \varepsilon_{y} \sigma_{s} \sigma_{p} .
$$

The partial emittance growth rates can be now written as follows:

$$
\begin{gathered}
\frac{d \sigma_{p}^{2}}{d t}=\frac{N r_{p}^{2} c L_{C}}{12 \pi \beta^{3} \gamma^{5} \sigma_{s}} \int_{0}^{L} \frac{d s}{L \sigma_{x} \sigma_{y}} S_{p}, \\
\frac{d \varepsilon_{y}}{d t}=\frac{N r_{p}^{2} c L_{C}}{12 \pi \beta^{3} \gamma^{5} \sigma_{s}} \int_{0}^{L} \frac{\beta_{y} d s}{L \sigma_{x} \sigma_{y}} \Psi\left(\frac{1}{\lambda_{1}}, \frac{1}{\lambda_{2}}, \frac{1}{\lambda_{3}}\right), \\
\frac{d \varepsilon_{x}}{d t}=\frac{N r_{p}^{2} c L_{C}}{12 \pi \beta^{3} \gamma^{5} \sigma_{s}} \\
\times \int_{0}^{L} \frac{\beta_{x} d s}{L \sigma_{x} \sigma_{y}}\left[S_{x}+\left(\frac{D_{x}^{2}}{\beta_{x}^{2}}+\Phi^{2}\right) S_{p}+S_{x p}\right],
\end{gathered}
$$

where functions $S_{p}, S_{x}$, and $S_{x p}$ are expressed through the functions defined in Eqs. (25)-(27) as 


$$
\begin{aligned}
S_{p}= & \frac{\gamma^{2}}{2}\left[2 R_{1}-R_{2}\left(1-\frac{3 a_{2}}{\sqrt{a_{2}^{2}+\gamma^{2} a_{x}^{2} \Phi^{2}}}\right)\right. \\
- & \left.R_{3}\left(1+\frac{3 a_{2}}{\sqrt{a_{2}^{2}+\gamma^{2} a_{x}^{2} \Phi^{2}}}\right)\right], \\
S_{x}= & \frac{1}{2}\left[2 R_{1}-R_{2}\left(1+\frac{3 a_{2}}{\sqrt{a_{2}{ }^{2}+\gamma^{2} a_{x}{ }^{2} \Phi^{2}}}\right)\right. \\
& \left.-R_{3}\left(1-\frac{3 a_{2}}{\sqrt{a_{2}{ }^{2}+\gamma^{2} a_{x}^{2} \Phi^{2}}}\right)\right], \\
S_{x p} & =\frac{3 \gamma^{2} \Phi^{2} a_{x}}{\sqrt{a_{2}{ }^{2}+\gamma^{2} a_{x}{ }^{2} \Phi^{2}}}\left[R_{3}-R_{2}\right] .
\end{aligned}
$$

Thus, by computing the three integrals $R_{1}, R_{2}$, and $R_{3}$ one fully defines the IBS rates at a given lattice location.

\section{SMOOTH APPROXIMATION}

In a smooth-lattice approximation case I will assume $\Phi=0$, thus

$$
\begin{gathered}
\lambda_{1}=a_{y}, \\
\lambda_{2}=a_{x}, \\
\lambda_{3}=\gamma^{2} a_{s} .
\end{gathered}
$$

The functions $S_{p}, S_{x}$, and $S_{x p}$ can be written as

$$
\begin{gathered}
S_{p}=\Psi\left(\frac{1}{\lambda_{3}}, \frac{1}{\lambda_{1}}, \frac{1}{\lambda_{2}}\right), \\
S_{x}=\Psi\left(\frac{1}{\lambda_{2}}, \frac{1}{\lambda_{3}}, \frac{1}{\lambda_{1}}\right), \\
S_{x p}=0 .
\end{gathered}
$$

These formulas can be simplified even further if one neglects the dispersion function, $D_{x}$. With this assumption, one obtains the following partial growth rates:

$$
\begin{gathered}
\frac{d \sigma_{p}^{2}}{d t}=\frac{N r_{p}^{2} c L_{C}}{12 \pi \beta^{3} \gamma^{3} \sigma_{s}} \int_{0}^{L} \frac{d s}{L \sigma_{x} \sigma_{y}} \Psi\left(\frac{\sigma_{p}^{2}}{\gamma^{2}}, \theta_{y}{ }^{2}, \theta_{x}^{2}\right) \\
\frac{d \varepsilon_{y}}{d t}=\frac{N r_{p}{ }^{2} c L_{C}}{12 \pi \beta^{3} \gamma^{5} \sigma_{s}} \int_{0}^{L} \frac{\beta_{y} d s}{L \sigma_{x} \sigma_{y}} \Psi\left(\theta_{y}^{2}, \theta_{x}^{2}, \frac{\sigma_{p}^{2}}{\gamma^{2}}\right) \\
\frac{d \varepsilon_{x}}{d t}=\frac{N r_{p}^{2} c L_{C}}{12 \pi \beta^{3} \gamma^{5} \sigma_{s}} \int_{0}^{L} \frac{\beta_{x} d s}{L \sigma_{x} \sigma_{y}} \Psi\left(\theta_{x}{ }^{2}, \frac{\sigma_{p}^{2}}{\gamma^{2}}, \theta_{x}^{2}\right)
\end{gathered}
$$

\section{ROUND-BEAM APPROXIMATION}

Further useful approximation can be obtained by assuming equal transverse beam sizes $\left(\sigma_{x}=\sigma_{y}=\sigma\right)$ and temperatures $\left(\theta_{x}{ }^{2}=\theta_{y}{ }^{2}=\theta^{2}\right)$. One can then introduce a single variable, $z$, such that

$$
z=\frac{\sigma_{p}^{2}}{\gamma^{2} \theta^{2}}
$$

is the ratio of the longitudinal to transverse temperatures in the beam rest frame. The partial IBS growth rates are expressed as follows:

$$
\begin{gathered}
\frac{d \sigma_{p}^{2}}{d t}=\frac{N r_{p}{ }^{2} c L_{C}}{8 \beta^{3} \gamma^{3} \sigma_{s} \sigma^{2} \theta} F(z), \\
\frac{d \varepsilon_{x, y}}{d t}=-\frac{N r_{p}^{2} c L_{C}}{16 \beta^{3} \gamma^{5} \sigma_{s} \varepsilon_{x, y} \theta} F(z),
\end{gathered}
$$

where

$$
F(z)=\frac{2}{3 \pi} \Psi(z, 1,1) .
$$

The function $F(z)$ can be expressed analytically through hyperbolic functions, instead I propose an approximate function, $G(z)$, which deviates from function $F(z)$ by no more than $20 \%$ in the range of $z$ values from 0 to 10 .

$$
\frac{2}{3 \pi} \Psi(z, 1,1) \approx G(z)=\left(1-z^{1 / 4}\right) \frac{\ln (z+1)}{z} .
$$

Both of these functions are shown in Fig. 1.

The round-beam approximation, presented here, is identical to that, obtained from the theory of Ichimaru and Rosenbluth [6] for a nonrelativistic plasma with initially unequal longitudinal and transverse temperatures. This theory has been recently confirmed by experiments [7] with a non-neutral electron plasma.

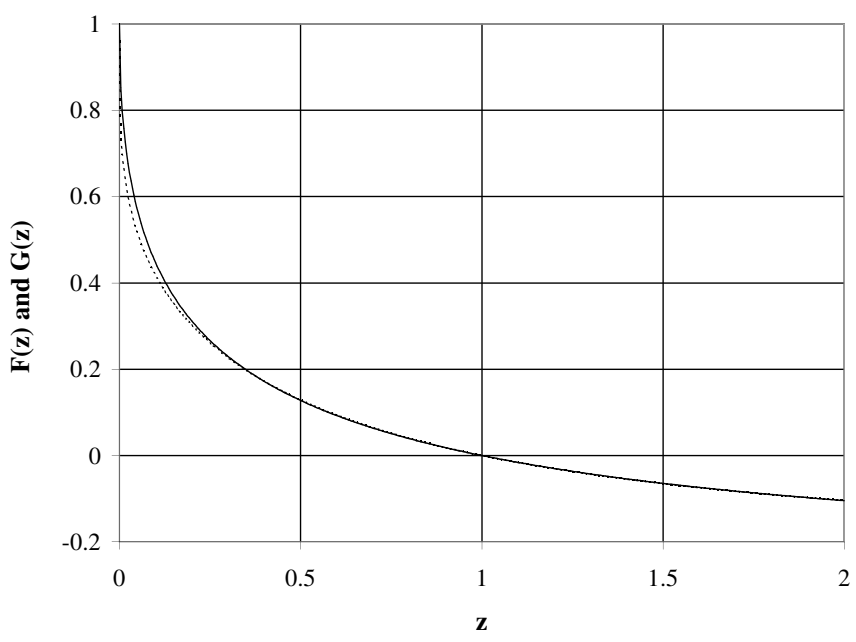

FIG. 1. The exact $[F(z)$, solid line $]$ and the approximate $[G(z)$, dashed line] IBS functions for a round beam. 
In a general case of unequal temperatures, if $T_{x}, T_{y}$, and $T_{z}$ are the plasma electron temperatures, the plasma temperature relaxation rates can be written as

$$
\frac{d T_{x}}{d t}=\frac{4 \sqrt{\pi} L_{C}}{3} n r_{e} c \sqrt{\left(m c^{2}\right)^{3}} \Psi\left(T_{x}, T_{y}, T_{z}\right),
$$

where $n$ is the plasma density, ions have been treated as a stationary background and the external fields have been neglected. Rates for $T_{y}$ and $T_{z}$ are obtained by cycling the variables in Eq. (50).

\section{COASTING BEAM IBS RATES}

The total 6-dimensional emittance growth rate for a coasting beam can be written as

$$
\begin{aligned}
\frac{1}{\tau} \equiv & \frac{1}{\Gamma} \frac{d \Gamma}{d t}=\frac{1}{\varepsilon_{x}} \frac{d \varepsilon_{x}}{d t}+\frac{1}{\varepsilon_{y}} \frac{d \varepsilon_{y}}{d t}+\frac{1}{2 \sigma_{p}{ }^{2}} \frac{d \sigma_{p}{ }^{2}}{d t} \\
= & \frac{N r_{p}{ }^{2} c L_{C}}{6 \sqrt{\pi} \beta^{3} \gamma^{5} L} \int_{0}^{L} \frac{d s}{L \sigma_{x} \sigma_{y}}\left[\lambda_{1} \Psi\left(\frac{1}{\lambda_{1}}, \frac{1}{\lambda_{2}}, \frac{1}{\lambda_{3}}\right)\right. \\
& + \text { two cyclic permutations }] .
\end{aligned}
$$

The partial emittance growth rates can be now written as follows:

$$
\begin{gathered}
\frac{d \sigma_{p}^{2}}{d t}=\frac{N r_{p}^{2} c L_{C}}{3 \sqrt{\pi} \beta^{3} \gamma^{5} \sigma_{s}} \int_{0}^{L} \frac{d s}{L \sigma_{x} \sigma_{y}} S_{p}, \\
\frac{d \varepsilon_{y}}{d t}=\frac{N r_{p}^{2} c L_{C}}{6 \sqrt{\pi} \beta^{3} \gamma^{5} \sigma_{s}} \int_{0}^{L} \frac{\beta_{y} d s}{L \sigma_{x} \sigma_{y}} \Psi\left(\frac{1}{\lambda_{1}}, \frac{1}{\lambda_{2}}, \frac{1}{\lambda_{3}}\right), \\
\frac{d \varepsilon_{x}}{d t}=\frac{N r_{p}^{2} c L_{C}}{6 \sqrt{\pi} \beta^{3} \gamma^{5} \sigma_{s}} \\
\times \int_{0}^{L} \frac{\beta_{x} d s}{L \sigma_{x} \sigma_{y}}\left[S_{x}+\left(\frac{D_{x}^{2}}{\beta_{x}^{2}}+\Phi^{2}\right) S_{p}+S_{x p}\right],
\end{gathered}
$$

where functions $S_{p}, S_{x}$, and $S_{x p}$ are defined in Eqs. (33)(35).

\section{CONCLUSIONS}

Starting from results reported in Ref. [1], I have expressed the IBS rates in a convenient form containing symmetric elliptic integrals. These elliptic integrals can be evaluated numerically by an efficient recursive procedure, described in Ref. [5]. The values of three elliptic integrals are required for each lattice location; however, Eq. (6) allows to reduce the number of integrals to two. The use of elliptic integrals in itself does not speed up the calculation process, while the recursive computation procedure does. There is some evidence that the calculation speed with the software package MATHEMATICA, using the recursive method, is a factor of 20-25 higher than a direct use of integrals from Ref. [1].

\section{ACKNOWLEDGMENTS}

The author expresses his gratitude to Valeri Lebedev and Alexey Burov for many insightful and fruitful discussions.

[1] J. D. Bjorken and S. K. Mtingwa, Part. Accel. 13, 115 (1983). I believe there is a misprint in Eqs. (4.17) and (2.2f) of this reference. The correct expression is given by Eq. (29) of the present paper.

[2] N.S. Dikansky et al., GSI Report No. 97-07, 1997, Chap. 5. The rates reported in the present paper and in Ref. [1] are a factor of 2 lower than the rates reported in this reference.

[3] B. C. Carlson, J. Res. Natl. Inst. Stand. Technol. 107, 413 (2002), and references therein..

[4] B. C. Carlson, Numer. Math. 33, 1 (1979).

[5] W.H. Press, S. A. Teukolsky, W. T. Vetterling, and B. P. Flannery, Numerical Recipes in C: The Art of Scientific Computing (Cambridge University Press, Cambridge, United Kingdom, 1992), p. 261.

[6] S. Ichimaru and M. N. Rosenbluth, Phys. Fluids, 13, 2778 (1970).

[7] A. W. Hyatt, C. F. Driscoll, and J. H. Malmberg, Phys. Rev. Lett. 59, 2975 (1987). See also B. R. Beck, J. Fajans, and J. H. Malmberg, Phys. Plasmas 3, 1250 (1996). 